\title{
Posterior Fossa Tumors in Pediatric Age Group, an Epidemiological Study
}

\author{
Ziauddin $\mathbf{M}^{1}$, Arman $\mathrm{DM}^{2}$, Ekramullah $\mathrm{SM}^{3}$, Mukherjee $\mathrm{SK}^{4}$, Rahman $\mathbf{M}^{5}$, DAS $\mathbf{S}^{6}$, Moktadir MR
}

Conflict of interest: There is no conflict of interest relevant to this paper to disclose.

Funding Agency : was not funded by any institute or any group.

Contribution of Authors : Principal Investigator- Dr. Md. Ziauddin

Manuscript preparation-Dr. D. M. Arman, Prof. Sk. Md. Ekramullah

Data collection- Dr. Sudipta Kumer Mukherjee, Dr. Moshiur Rahman

Editorial formatting- Dr. Md. Ruhul Moktadir

Copyright: @2020bang.BJNS published by BSNS. This article is published under the creative commons CC-BY-NC license. This license permits use distribution (https://creativecommons. orgf/licences/by-nc/4-0/)reproduction in any medium, provided the original work is properly cited and is not used for commercial purposes.

Received: 15/10/2020

Accepted: 30/10/2020

\begin{abstract}
:
Background: There are no data so far relating posterior fossa brain tumors in pediatric age group in Bangladesh. We are dealing with this type of tumors regularly. So an attempt has been made to document these tumors in respect to their incidence in our country, mode of presentation, age and sex distribution, treatment offered to them and their complication and outcome.
\end{abstract}

Methods: This study was conducted in the Department of Pediatric Neurosurgery, National Institute of Neurosciences and Hospital. It was a retrospective study and cases were collected from 2013-2020.

Result: We dealt with 60 cases of posterior fossa brain tumors. Out of them 21 were medulloblastoma, 19 pilocytic astrocytoma, 11 ependymoma and rest are other tumors. Almost all patients presented with hydrocephalus and in most cases CSF diversion was done before definitive tumors surgery.

Conclusion: This is only a partial picture of total posterior fossa tumors in pediatric age group. This type of study should be done in a larger scale and newer modalities of diagnostics should be widely practiced for a better post-operative treatment and better prognosis.

Key words:Posterior fossa tumor, Pediatric, Epidemiological.

Bang. J Neurosurgery 2021; 10(2): 182-184

\section{Intrduction:}

Brain tumors are the second most common neoplasm and the most common solid tumor in children. They are the third leading cause of death in children younger than 16 years of age. ${ }^{1}$

Approximately $60 \%$ of these tumors occur below the tentorium, including the brainstem, cerebellum, fourth ventricle and cerebellopontine angle. The pathological features of these tumors are diverse. Prognosis ranges from excellent to dismal, depending on histopathological findings, extent of surgical resection and use of adjunctive therapies. ${ }^{2}$
Four tumor types comprise majority of the posterior fossa tumors- cerebellar astrocytoma, ependymoma, primitive neuroectodermal tumor/medulloblastoma and brainstem glioma. ${ }^{3}$

Signs and symptoms of increased intracranial pressure are the most common presentation of these tumours as they are usually sizable at presentation, causing obstructive hydrocephalus due to obstruction of the cerebrospinal fluid (CSF) pathway at the level of the fourth ventricle. ${ }^{4}$ They charactically present with symptoms and signs of hydrocephalus which include

1. Dr. Md. Ziauddin, Assistant Registrar, Department of Pediatric Neurosurgery, NINS.

2. Dr. D. M. Arman, Assistant Professor, Department of Pediatric Neurosurgery, NINS.

3. Prof. Sk. Md. Ekramullah, Professor and Head of Department of Pediatric Neurosurgery, NINS.

4. Dr. Sudipta Kumer Mukherjee, Associate Professor, Department of Pediatric Neurosurgery, NINS.

5. Dr. Moshiur Rahman, Medical Officer, Department of Pediatric Neurosurgery, NINS.

6. Dr. Subhasish Das, Assistant Professor, Department of Paediatrics, MAG Osmani Medical College.

7. Dr. Md. Ruhul Moktadir, Registrar, Department of Pediatric Neurosurgery, NINS.

Address of Correspondence: Dr. Md. Ziauddin, Assistant Registrar, Department of Pediatric Neurosurgery, NINS. Mobile: 01716078030, E-mail: drziahasnat@gmail.com 
progressively worsening morning headache and vomiting, followed by unsteadiness, double vision and papilledema. $^{5}$

The overall 5-year survival rate of children with brain tumors has improved considerably over the past several years. Because of earlier diagnosis and better therapies, survival rates are now between $35 \%$ and $65 \%$, depending on several factors, including tumor histology and location. Age is also an important prognostic indicator for children with CNS tumors in general. Data consistently show that 10 - to 15 -yearolds have the longest survival whereas those younger than 2 years have the shortest. ${ }^{6}$

Great technological strides have been made in regard to improving and understanding tumor biology, imaging, surgical techniques and chemotherapeutic/ radiation protocols, leading to increased survival time in these patients. However, these treatments can lead to significant morbidity to the developing brain and thus we still have more to learn from these complex and challenging tumors. Infratentorial(posterior fossa) brain tumors $(60 \%)$ are:

1. Medulloblastoma

2. Brainstem glioma

3. PCA of cerebellum

4. Ependymoma

5. Dermoid

6. Epidermoid

7. Vestibular Schwannoma

8. Atypical teratoid/ rhabdoid

9. Choroid plexus papilloma. ${ }^{7}$

\section{Materials and methods:}

This is an observational study carried out in the Department of Pediatric Neurosurgery, National Institute of Neurosciences and Hospital. It was a retrospective study and cases were collected from 2013-2020. This study includes 60 patients. Most of the patients presented to us with headache and vomiting. Other symptoms were gait disturbance, visual disturbances, cognitive impairment and convulsion. After clinical assessment we usually first do a CT scan of brain and mark it as PFSOL with or without hydrocephalus. Then MRI with contrast is done, followed by visual assessment study including color fundus photograph to check the urgency of doing CSF diversion by seeing papilledema grade.

After proper evaluation surgery is done by either craniotomy or craniectomy. Usually if tumor is found solid and more vascular then we do craniectomy and if tumor is mostly cystic and less vascular we prefer craniotomy. If vision is in danger we often do CSF diversion before definitive surgery. Four histopathological samples are made, two sent immediately for histopathological confirmation of tumor type and other two kept to clear if any confusion about diagnosis or further immunohistochemical analysis needed. After tumor removal water-tight dural closure done by usualy by using partial thickness cervical fascial graft. Then rest of the incision closed in multilayer without using any drain.

\section{Results:}

We dealt with 60 cases of posterior fossa brain tumors. 22 of them were within 0-5 years age group, 18 were within 6-10 years age group and 20 were within 11-15 years age group. No posterior fossa tumor were found with age 16-18 years.

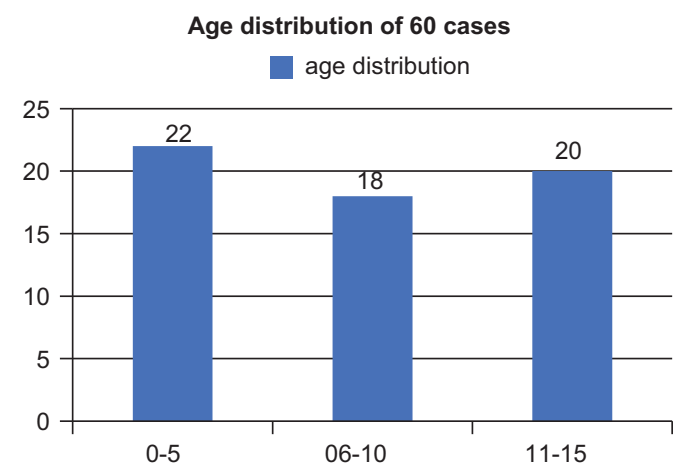

Fig-1: Age distribution of posterior fossa tumors of 60 patients

Most the patients were male in our series and which is $36(60 \%)$.

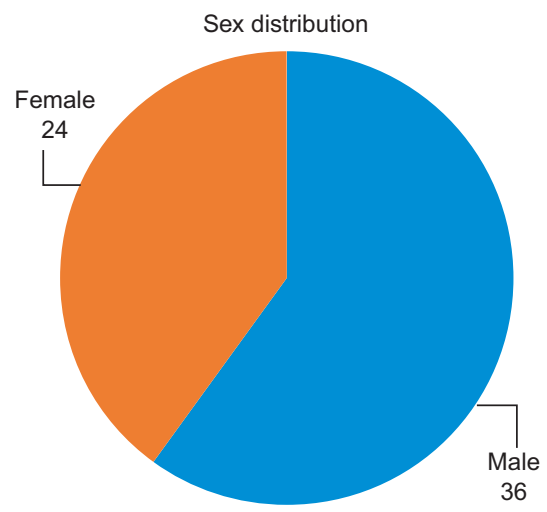

Fig.-2: Sex distribution of pediatric patients with posterior fossa tumors 
Out of them 21 were medulloblastoma, 19 pilocytic astrocytoma, 11 ependymoma and rest are other tumors. Almost all patient presented with hydrocephalus and in most cases CSF diversion was done before definitive tumors surgery.

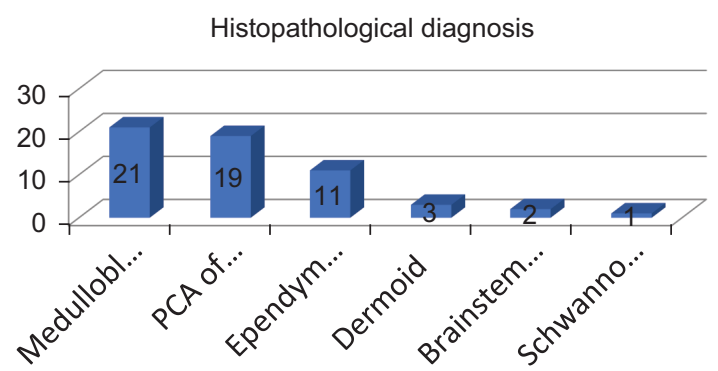

Fig.-3: Histopathological diagnosis of patients with posterior fossa tumors in pediatric age group

\section{Discussion:}

More boys than girls are affected depending on tumor type and patient age. ${ }^{8}$ Among all 60 patients most were $(60 \%)$ male, so PFSOL in pediatric age group in Bangladesh is mainly male predominant which correlated with international data.

They are almost equally distributed among 0-5, 6-10 and $11-15$ years of age group with $0-5$ years being the most $(37 \%)$ followed by $11-15$ years $(33 \%)$ and $6-10$ years $(30 \%)$. Another trend is observed that with $0-5$ years most histopathological diagnoses are medulloblastoma and in 11-15 years most are pilocytic astrocytoma.Bonner et.al.in n1988 found that 40\% of medulloblastoma present in their first 5 years of life and $75 \%$ in their first decade. ${ }^{3}$

The relative incidence of posterior fossa neoplasms in pediatric population is medulloblastoma $50 \%$, cerebellar astrocytoma $30-40 \%$, ependymoma 10 $20 \%$ brainstem glioma $10-20 \%$. Our histopathological analysis shows 21 out of 60 patients were medulloblastoma(35\%), followed by 19 pilocyticatrocytoma(32\%) and 11 ependymoma(18\%), 3 dermoid( $5 \%$ ) and others like brainstem glioma, pineoblastoma, germinoma in very few numbers.'

Most of the patient with PFSOL presented with hydrocephalus and it is $88 \%$ of all cases of which is similar to the findings of Lin CT et. al. which says about $70-90 \%$ patient with posterior fossa present with hydrocephalus. Due to huge workload and unavoidable delay in definitive surgery we often do preoperative CSF diversion to save the vision. The options are mainly VP shunt or endoscopic third ventriculostomy.
It seems to diminish the rate of perioperative complications and persistent hydrocephalus after tumor resection. On the other hand, the rate of persistent hydrocephalus after tumor resection is 10$30 \%$ only and an ETVin every patient prior toposterior fossa tumor surgery would lead to an unnecessary surgical procedure in at least $70-90 \%$ of them. ${ }^{9}$

Most of the time we can do gross total resection ,but sometimes neartotal or subtotal resection is also done. Post operative CT scan is done in $1^{\text {st }}$ POD. Surgery is followed by radiotherapy and chemotherapy as needed. Patient is usually followed up at $1 \mathrm{~m}$ onth after discharge, then 6 monthly for 1 year and then yearly later on. During follow up CT or MRI scan with contrast is done after patient assessed clinically.

\section{Conclusion:}

This is only a partial picture of total posterior fossa tumors in pediatric age group. This type of study should be done in a larger scale and newer modalities of diagnostics should be widely practiced for a better post-operative treatment and better prognosis.

\section{References:}

1. Shiminski-Maher T, Shields M. Pediatric brain tumors: diagnosis and management. Journal of Pediatric Oncology Nursing. 1995 Oct 1;12(4):188-98.

2. Weeks A, Fallah A, Rutka JT. Posterior Fossa and Brainstem Tumors in Children. In Principles of Neurological Surgery 2012 Jan 1 (pp. 169-185). WB Saunders.

3. Bonner K, Siegel KR. Pathology, treatment and management of posterior fossa brain tumors in childhood. The Journal of Neuroscience Nursing: Journal of the American Association of Neuroscience Nurses. $1988 \mathrm{Apr}$ 1;20(2):84-93

4. Rasalkar DD, Chu WC, Paunipagar BK, Cheng FW, Li CK. Paediatric intra-axial posterior fossa tumours: pictorial review. Postgraduate medical journal. 2013 Jan 1;89(1047):39-46.

5. Albright AL. Pediatric brain tumors. CA: a cancer journal for clinicians. 1993;43(5):272-88.

6. Winn HR. Youmans Neurological Surgery E-Book. Elsevier Health Sciences; 2011 Nov 17.

7. Ellenbogen RG, Sekhar LN, Kitchen N. Principles of Neurological Surgery E-Book. Elsevier Health Sciences; 2017 Dec 13.

8. Kakar J, Ashraf J, Khan AA, Imran M, Rehmani MA, Ghori SA, Ali MF. The satisfactory surgical outcome of posterior fossa brain tumors in children at civil hospital, karachi. Asian Journal of Neurosurgery. 2020 Apr 1;15(2):377.

9. Marx S, Reinfelder M, Matthes M, Schroeder HW, Baldauf J. Frequency and treatment of hydrocephalus prior to and after posterior fossa tumor surgery in adult patients. ActaNeurochirurgica. 2018 May 1;160(5):1063-71.

10. Lin CT, Riva-Cambrin JK. Management of posterior fossa tumors and hydrocephalus in children: a review. Child's Nervous System. 2015 Oct 1;31(10):1781-9. 